\title{
Enhancing Enterprise Information Systems Synergy in Disaster Management
}

\author{
Ovidiu Noran \\ Griffith University, Nathan QLD 4111, Australia \\ O.Noran@griffith.edu.au
}

Keywords: Disaster Management, Enterprise Architecture, Collaborative Networks.

\begin{abstract}
Climate change-triggered catastrophic events appear to be steadily increasing in intensity and frequency. Proper preparation, response and recovery are essential in order to survive and recover from disasters. Unfortunately however, the organizations responsible for delivering emergency response services often underperform, mainly owing to the lack of proper collaboration (especially interoperation) of their information systems. This paper analyses disaster management-specific collaboration issues from an enterprise information systems perspective and proposes improvements based on advances in information systems and interoperability research, using an enterprise architecture perspective and artefacts in order to provide a sustainable holistic and life cycle-based solution.
\end{abstract}

\section{INTRODUCTION}

Although emphasis on environmental sustainability and emissions reduction is increasing worldwide, any significant results are bound to take extensive time to slow down and reverse the current trend of environmental degradation. In the meantime, climate change-triggered catastrophic events appear to steadily increase in intensity and frequency. In this context, it is essential to be able to promptly and effectively prevent, prepare for, respond to and recover from catastrophic events. Governments worldwide usually react by creating policies and organisations (e.g. agencies) to tackle these aspects. The 'disaster management organisations' (DMOs) thus created operate in a complex environment (history / tradition, geographic location and culture, level / type of governance etc), that typically triggers heterogeneity and independent evolution. The resulting organisational diversity, while generally beneficial, requires additional effort to achieve proper and effective collaboration (Whitman and Panetto, 2006). As coping with large scale catastrophic events typically requires resources and capabilities beyond those of individual organisations, effective cooperation of DMOs at all necessary levels and addressing all relevant aspects is essential. Failing to achieve this has dire consequences including loss of property and human life.

Enterprise Information Systems (EISs), as an es sential and ubiquitous component of every organisation (Lehtinen and Lyytinen, 1986), play an essential role in achieving the level of cooperation required to enable organisational sustainability of the DM endeavour. This paper aims to focus on the information system (IS) aspect of disaster management interoperability (as a crucial component of cooperation) and to propose ways to address them using knowledge accumulated in the IS and interoperability research areas. The analysis is performed from an enterprise architecture (EA) point of view and using EA artefacts, in an attempt to promote a whole-system and life cycle-based approach covering all aspects deemed as relevant for the DM universe of discourse.

\section{DISASTER MANAGEMENT: CURRENT ISSUES}

The operation of emergency services is typically regulated at state, national and international levels (see (Australian Government, 2011); (Federal Emergency Management Agency, 2011); (Go-vernment of South Australia, 2004); (United Nations International Strategy for Disaster Reduction Secretariat (UNISDR), 2011)). However, simply instructing organisations to cooperate using generic directives does not bring about true collaboration. The likely 
consequences are increased response times, confusion about the situation on the ground and dispute as to who, where and when is in charge.

Often, it is also difficult to coordinate the EISs of DM participants due to incompatibilities in infrastructure and difficulty in filtering and validating the typical flood of information generated during disaster events. For example, inconsistency in the type and format of alert messages may delay intervention and hinder warnings by creating a situation where the population is 'intoxicated' with numerous ambiguous and locally-irrelevant messages (Queensland Floods Commission of Enquiry, 2011); (Victorian Bushfires Royal Commission, 2009). This may lead to reduced prevention and response efforts on the part of the intended recipients, potentially resulting in property and life loss situations. Currently, efforts to standardise warning message protocols are localised and enjoy rather low take-up rates (OASIS, 2005).

Two main approaches have been traditionally used to address DMO cooperation problems. They involve either centralisation (hierarchical command) or federalisation of emergency services. Irrespective of the approach used, proper emergency response and cooperation has still not been achieved, as reflected in criticism expressed in various literature (e.g. ('t Hart et al., 2005); (Clark, 2006); (Wiese, 2006)). The use of military operations-style network-enabled capabilities as the backbone of disaster management (von Lubitz et al., 2008) is valid only as part of the overall DM effort; in addition, it also risks leading to over-reliance on infrastructure that is very likely to fail during disaster events.

Various documents, inquiries reviews and reports (('t Hart et al., 2005); (Brewin, 2011); (Igarashi et al., 2011); (Queensland Floods Commission of Enquiry, 2011); (United Nations International Strategy for Disaster Reduction Secretariat (UNISDR), 2011); (Victorian Bushfires Royal Commission, 2009); (Wiese, 2006), etc), suggest that the culprits responsible for the current shortcomings could in fact be the inadequate information and knowledge flow and quality between the participants' EISs (Prizzia and Helfand, 2001); (Wickramasinghe and von Lubitz, 2007). The main causes of these problems appear to be heterogeneity-induced lack of compatibility, mistrust, organisational confusion (who does what and when) and even perceptions of competition. True and efficient collaboration requires the interoperability of processes, resources and organisational cultures of the participants (Kapucu et al., 2010); (Trakas, 2012), all of which are reflected in their EISs (Stohr and Konsynsky,
1992). Another set of essential aspects addressed to a lesser extent are the life cycles of the DMOs, disaster management task forces (DMTFs), government agencies, legislation, service providers, disaster events and especially the relations between these life cycles.

Other domains have also struggled with such problems and have come up with possible solutions. For example, the DMOs' situation resembles that of commercial enterprises that need to take on projects beyond their own resources and knowledge, in the context of global economy. Their solution is to set up or join so-called 'Collaborative Networks' (CNs) (Camarinha-Matos et al., 2009). Another valid analogy is that of allied armed forces that prepare to cooperate in crisis situations by using standardised agreements and joint exercises (NATO, 2006).

As DMOs worldwide may have full time, militarised and voluntary / reserve staff components (depending on the geographical location and local legal and administrative situation), concepts from the above commercial and military areas can be used (provided of course that proper customisation is performed so as to match the specific scenario).

\section{DISASTER MANAGEMENT COOPERATION: APPROACH AND ASPECTS}

DM cooperation involves many aspects; for example, in addition to receiving the mandate to cooperate, DMOs must also have the will and especially the capability to work together in an optimal way.

The concept of interoperability is often used as a measure of IS cooperation capability (e.g. the Levels of Information System Interoperability (LISI) taxonomy in the Department of Defence Architecture Framework v1 (2004)).

The analysis of interoperability in the DM domain must include some important aspects:

- what is the required interoperability extent?

- what components and / or aspects need to interoperate?

- how can it be ensured that all necessary aspects are covered?

- can sustainable interoperability be achieved as all participants evolve?

\subsection{Extent of Interoperability}

As each disaster event is unique, there is no 'one 
size fits all crisis situations' DMO interoperability level. At a minimum, the participating organisations' systems should be compatible, so they don't hinder each other's operations (see Fig. 1).
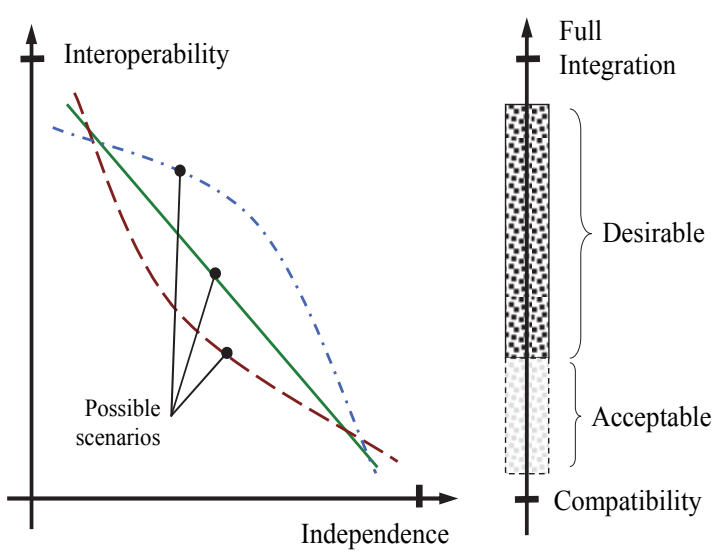

Figure 1: Acceptable and desirable EIS Interoperability levels in disaster management.

In an emergency situation, the EISs of some task force members are likely to be affected to various degrees (culminating in complete shutdown). In this situation, the remaining DMOs should be able to continue within acceptable performance parameters (see e.g. the ARPANET resilient network concept (Heart et al., 1978)) and ideally, compensate for the essential duties of the failed participants.

In a different scenario, even if the central point of command and task force members were not affected by the disaster event(s), the coordination between them could still be severely hindered by communication infrastructure breakdown (see for example (Crawford, 2012); (Queensland Floods Commission of Enquiry, 2011)). In this situation, the task force participants should be able to autonomously carry on their duties for a certain amount of time. Such a capability can be acquired based on pre-agreed procedures and shared knowledge set up in advance and continuously monitored and updated to maintain relevance and consistency.

Reviewing the relevant interoperability body of knowledge we find that ISO14258 (ISO, 2005) establishes several ways to achieve interoperability: integrated (common format for all models), unified (common format at meta level) and federated (participants negotiating an ontology as they go to achieve a shared understanding of models). In the case of DMOs, neither full EIS integration nor federalisation appeared to achieve the desired results, mainly due to the organisational heterogeneity of DMOs and the impossibility to negotiate 'on the fly' during disaster event response.
The unified approach (who according to literature appears the most feasible in this situation) requires only the ontology to be negotiated in advance. Unfortunately however, despite significant ontology integration advances, currently in DM there seems to be no effective solution other than the DMOs spending time well before disaster events in order to agree on the meanings associated with the concepts used to exchange knowledge. Once that is achieved, proper EIS semantic interoperability should be almost intrinsic to the task forces formed by the participant DMOs.

\subsection{Interoperability Aspects}

Of all the interoperability aspects reviewed in the relevant literature, the most stringent in the disaster management area appear to be data and process. Thus, the ability to extract and exchange data from heterogeneous sources (delivering high volume and often unreliable data during disaster events) is paramount to being aware of the conditions on the ground and avoiding potentially life-threatening situations for emergency crews and population. Prior agreements on data format and especially on its meaning are essential. 'Process interoperability' in this context concerns the capability to perform joint operations but also to 'take over' and perform processes on behalf of a disaster management task force participant that may have been temporarily or permanently disabled.

The pragmatic interoperability aspect as described by Whitman and Panetto (2006) relates to the willingness and capacity of the participants to cooperate. In disaster management, the human component of the EIS needs attention prior to task force formation in order to allow gaining trust and common understanding among the participants.

Organisational interoperability is an important aspect in disaster management as task force participants may often exhibit significant organisational structure diversity. The issues identified by Chen (Chen, 2006) based on the Enterprise Interoperability Framework (EIF), namely responsibility, authority and type of organisation can all impact heavily on the functionality of the disaster management task force. In a crisis situation, the roles (mapping of the human resources onto the decisional structure) and hierarchy must be clear to everyone from the start so that the DMTF can 'hit the ground running' and focus straight on the disaster event rather than waste precious time figuring out its own management and operational details.

Finally, cultural interoperability (Whitman and 
Panetto, 2006) appears to be one of the hardest obstacles to overcome. The only current working solution appears to be regular immersion of the participant organisations in each other's cultures (for example, Army joint exercises, or expatriates as skilled language translators). This immersion facilitates the transfer and conversion of tacit and explicit knowledge between the participants and thus enables the unified approach previously identified as optimal in disaster management.

A recurring concept through all aspects analysed above is the 'co-habitation' of the organisations that are expected to form disaster management task forces, seen as a prerequisite towards the achievement of EIS interoperability - whether functional, informational, organisational or cultural. This paper proposes to tackle the co-habitation concept by adopting a life cycle-based, customised Collaborative Network paradigm.

\section{COLLABORATIVE NETWORKS FOR DISASTER MANAGEMENT}

Collaborative Networks $(\mathrm{CNs})$ are created in order to allow participating companies to know each other, gain trust and establish common agreed-upon procedures and infrastructure baselines. This enables CN members to promptly form Virtual Organisations (VOs), i.e. groups of companies that temporarily come together under a unique identity in order to bid for, win and complete projects requiring extensive resources and knowledge. A CN 'lead partner' may also be present, (self-)elected on size, influence, resources, etc. CN partners may take part in one or several VOs at any given time.

The CN concept may be adapted and applied to the disaster management area. Thus, the time available for the set-up of a 'disaster management' VO (here represented by the DMTF) is significantly shorter than that available for a commercial project bidding process. In addition, the disaster management CN (DMCN), its participants and the DMTF(s) produced will operate under tight legal operational guidelines set by relevant Governments and national and international frameworks (e.g. (Federal Emergency Management Agency, 2011).

The commercial and competitive motivations of the typical $\mathrm{CN}$ participants will translate in this case into efficiency and cooperation motivations reflected in lives and property rescued. The usual create / join / remain / leave the $\mathrm{CN}$ decision processes are man- dated from outside (or by the lead partner) for most participants. Reference models (patterns) classified on type and location (such as flooding, tornadoes, wildfires, earthquakes etc (Ellis et al., 2004)) are to be created from lessons learned in past disaster relief efforts and used to refine future DMTFs. shared repository and customised for specific geography, intensity, duration, side-effects and consequences.

The DMTF(s) produced by the DMCN will be set up for and assigned a specific DM project relating to a particular disaster event (or combination thereof). EIS cooperation aspects such as management, communication infrastructure, and other organisational interoperability issues would have been sorted out in advance within the DMCN, ensuring a prompt and appropriate task force response (addressing a typical shortcoming identified in past crisis management efforts (Trakas, 2012)).

Human-related aspects such as trust, organisational culture and recognition (featuring prominently in volunteer-based organisations (Esmond, 2011); (McLennan, 2008)) could be also addressed using the concept of 'emergency services academies' with local branches providing training based on a consistent interstate curriculum (see (Queensland Government, 2011) for an example).

\section{THE LIFE CYCLE CONTEXT: AN ENTERPRISE ARCHITECTURE VIEW}

All entities involved in disaster management efforts continuously change, going (sometimes repeatedly) through a set of life cycle phases that form the entities' 'life histories' (ISO/IEC, 2005). EIS interoperability requirements are inherently linked to life history as they will vary during each host organisation's life cycle phase; therefore, it is essential that the analysis and search for cooperateon and interoperability improvements is performed in a life cycle context.

In the following we argue that adopting an 'enterprise architecture' (EA) approach provides an optimal way to integrate the life cycle aspect into EIS cooperation analysis. This is because EA provides a holistic approach to business evolution and agility "by creating, communicating and improving the key requirements, principles and models that describe the enterprise's future state [...]. EA comprises people, processes, information and technology of the enterprise, and their relationships to one another and to the external environment" (Gartner Research, 2012). This EA definition is in agreement with the view of 


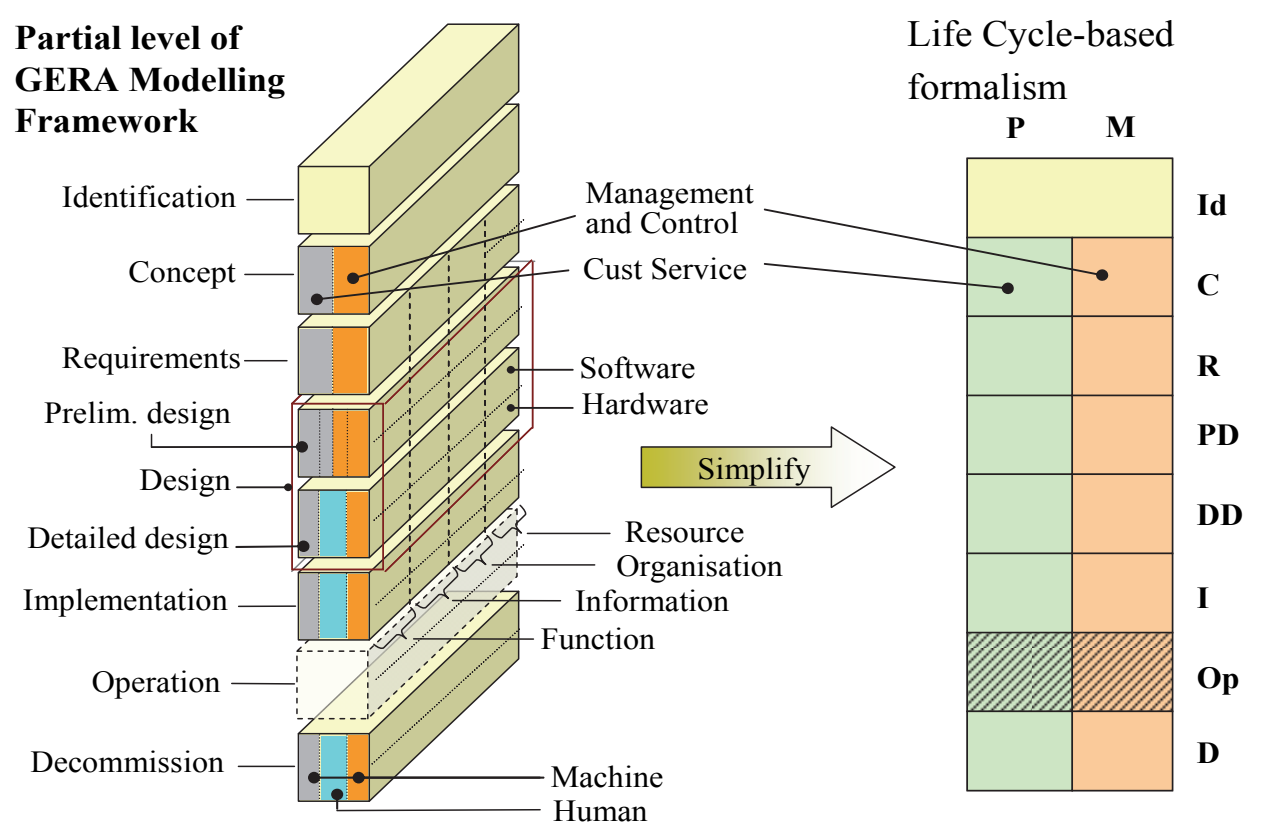

Figure 2: Using Enterprise Architecture Modelling Framework elements to develop a life cycle formalism.

IS as a socio-technical system (Pava, 1983) with voluntaristic people (McGregor, 1960) in a complex organisational, political and behavioural context (Iivari, 1991); (Keen and Morton, 1978). As such, it enables the use of EA as a life cycle-based, integrated and holistic approach to analyse the EIS cooperation requirements of the DMCN and DMTF participants.

To illustrate the way EA artefacts and methods can guide and enrich the analysis and improvement of DMO cooperation, we have selected a generic architecture framework (AF) that subsumes and abstracts several other mainstream AFs, defined in Annex A of ISO15704 (ISO/IEC, 2005) and called the Generalised Enterprise Architecture and Methodology (GERAM). The modelling framework (MF) of GERAM's reference architecture (called GERA) contains a rich repository of aspects (notably including human) that can all be represented in a life cycle context. GERA's MF has been used to model and analyse systems across many areas (e.g. (Mo, 2007); (Noran 2008; 2009)).

Aspect-based subsets of the GERA MF can be turned into life cycle-based formalisms used to produce business models requiring a life cycle-based analysis. For example, the aspects previously identified as significant in improving EIS cooperation in disaster management (such as function, information, resources, organisation) but also additional useful viewpoints like management vs. operations, automation boundary / human extent, etc) can be represented as shown in Fig. 2, left.
Aspects can also be separated to promote clarity by using 'flattened' representations; for example, the 2-dimensional structure shown in Fig. 2 right is used to focus on the product/service and management viewpoints in a life cycle context. This formalism can then be used for separate, dedicated function, information (see Fig. 5), resource, organisation, hardware, software etc diagrams.

Fig. 3 left shows the use of the above-described formalism to represent a disaster event, focusing only on the relevant life cycle phases and relations to other events' life cycles. Thus, Fig. 3 left shows how a Primary Disaster Event (PDE) can trigger / influence other events (SDE, TDE); For example, an earthquake PDE can trigger a tsunami SDE that can in turn trigger a partial nuclear meltdown TDE. The model also shows that PDE can also influence TDE directly and/or in parallel. Events such as chain reactions can also be shown (arrows from Operation to Implementation within the same entity). Note that here, the meaning of the generic GERA MF 'Detailed Design' and 'Implementation' life cycle phases refers to features of the event - e.g. earthquake time, epicentre and duration or tsunami spread, wave speed, height etc.

The right hand side of Fig. 3 shows how the life cycle phases of a disaster management project (DMP) can be mapped against typical disaster management activities (e.g. as defined by the Australian Government (2011)) performed by a DMTF that sets up and operates that project. 
Disaster Event modelling

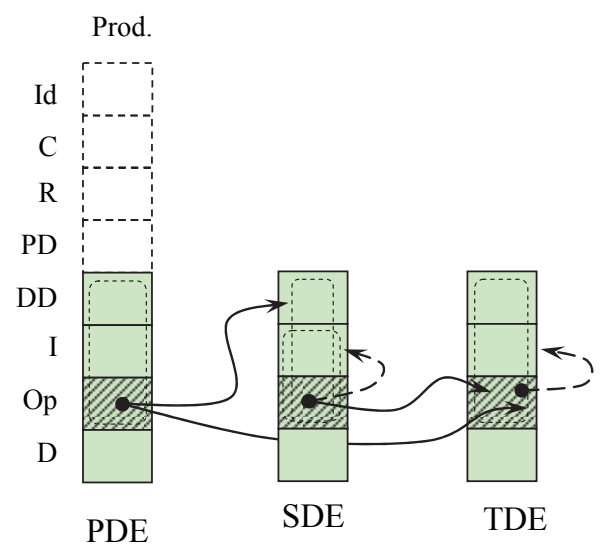

Disaster Event mapping

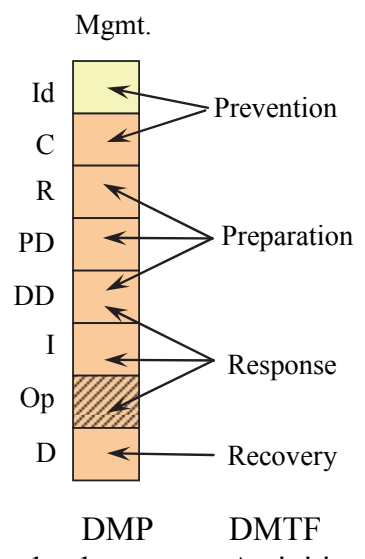

life cycle phases Activities

Legend:

PDE / SDE / TDE = Primary / Secondary / Tertiary Disaster Event;

DMP $=$ Disaster Mgmt Project; DMTF = Disaster Management Task Force

$\square$ = Production view $\square=$ Management view $\bullet-\rightarrow=$ Possible scenario

Figure 3: Disaster events mapping and modelling accomplished using a life cycle-based modelling formalism.

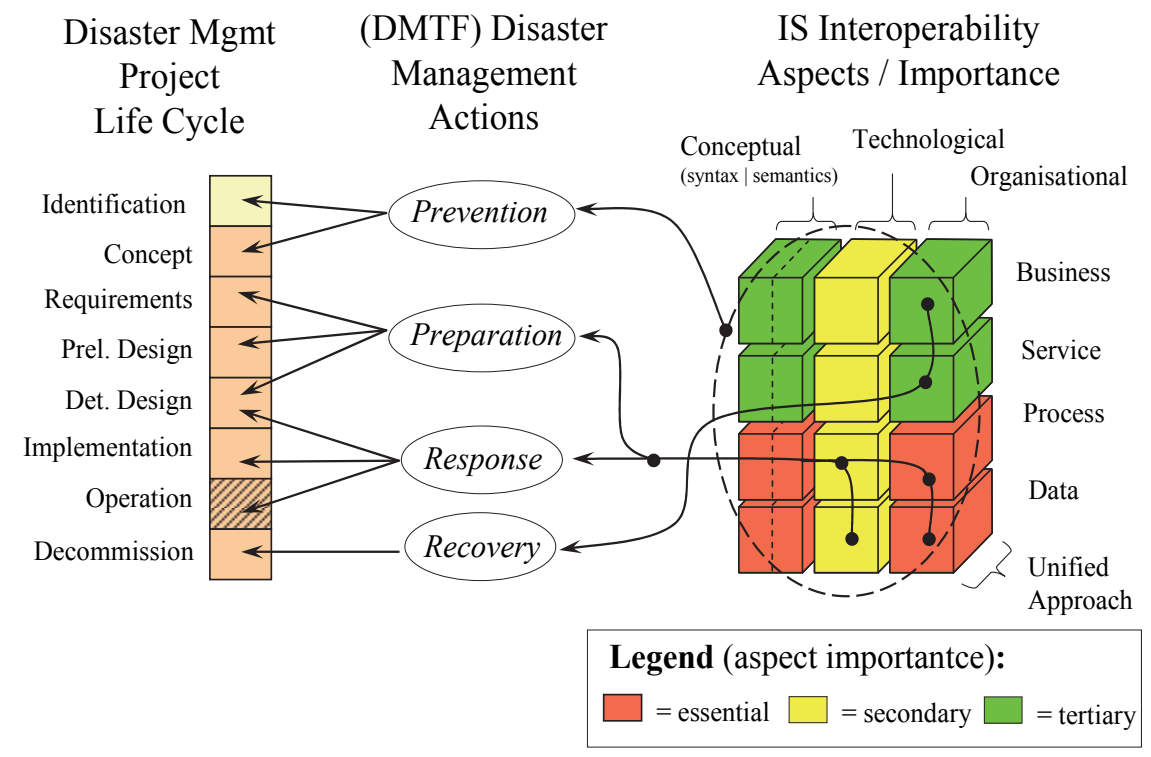

Figure 4: Enterprise Information Systerms' Interoperability requirements mapped on disaster management actions and the disaster management project life cycle, using Chen's EIF (Chen, 2006) and GERA's MF-based formalism.

Such diagrams are useful to help DMCN and DTMF participants to promptly grasp a common understanding of the disaster event lifecycle and management aspects, thus overcoming initial EIS interoperability semantic barriers and facilitate all DMP phases Fig. 4 uses a modified version of Chen's EIF and GERA's MF-based life cycle modelling formalism to show how the relevance and applicability of the EIS interoperability aspects depend on the specific life cycle phases of the disaster event and the DMTFs' actions to address them.

As can be seen from the figure, various interoperability aspects may take precedence as the disaster event life cycle phases unfold. For example, during the response phase, data and process IS interoperability aspects are paramount. This is because accurate and fresh data is required and processes may need to be performed interchangeably due to poten- 
tial disability of some DMTF participants (or the need to call upon replacement DMCN members). During the recovery, which typically takes place over an extended period of time and requires less real-time responsiveness, organisational business and service EIS interoperability aspects may become more important.

\section{SAMPLE APPLICATION TO EIS INTEROPERABILITY}

Fig. 4 shows only the influence of the disaster event, management project and task force life cycles on the interoperability requirements. As a matter of fact, DMCNs, DMOs, DMTFs and DMPs interact with a plethora of entities and artefacts during their life history. These interactions must also be analysed in order to gain a holistic view of the EIS cooperation requirements.

Using the GERA-based formalism described in Section 5, diagrams can be constructed for the aspects identified in Section 3.2 as relevant to IS interoperability. For example, Fig. 5 shows interoperability-related interactions for the Information (data) interoperability aspect, in the previously described scenario of a collaborative network (DMCN) creating task forces (DMTFs) supervising disaster management projects (DMPs) that coordinate disaster event responses.

The arrows in the figure show data interoperability requirements and influences. For example, the participating DMOs' EISs need to interoperate during their Operation life cycle phase; however this may also imply some redesign (line between DMO1 and DMO2 linking Operation and other life cycle phases, depending on the redesign extent). The EIS of the DMBE must be able to interoperate with the EIS of the DMTF it creates and DMPs managed by them in their Operation life cycle phases, with influences on other phases in case of subsequent redesign (details omitted for clarity).

In another example, data 'interoperation' (i.e. properly interpreting the messages) of the population POP with the Government's Disaster Management Agencies (GDMAs, such as (Government of Queensland, 2012)) is important as may result in changes to legislation - hence in the DMO and DMBE interoperability requirements. However, data interoperability between the EISs of DMOs participating in a DMTF and between the DMTF's EIS and POP is paramount as it will directly influence the extent of lost property and casualties. Past experience and feedback from disaster enquiries (Ellis et al.,
2004); (Queensland Floods Commission of Enquiry, 2011); (Victorian Bushfires Royal Commission, 2009) has shown that two major goals of the disaster management cooperation enhancement effort (partly reflected in Fig. 5) should be: a) whether POP receives, understands, believes and acts on DMTF warnings and directives and b) that DMTF participants can properly interoperate during the disaster event. Thus the EIS of the DMTFs (and implicitly DMOs) should be also designed to avoid ambiguity and maximise focus in relation to the local specific semantic interoperability requirements (language, technology type, customs etc). For example, the message format and distribution in a densely populated and developed area would most likely differ from the one used in a sparsely and/or underdeveloped region - at least until warning information standardisation is successfully and widely implemented.

Importantly, some organisations shown in Fig. 5 should be able to redesign themselves to a certain extent (arrows from Operation life cycle phase to upper phases within same entities e.g. in DMBE, DMO, DMTF). This reflects an essential capability to adapt (and thus be agile and resilient) in the face of changes in the situation and environment that are likely to occur briskly and unexpectedly during disaster events.

The Disaster Management Qualification and Training organisation (DMQT, e.g. (Queensland Government, 2011)) can assess the suitability of organisations to enter a DMBE by requiring (and providing training if appropriate) EIS data interoperability between DMO applicants and the DMBE. This requirement may also go beyond the Operation life cycle phase, should training / redesign of the participants be performed.

The validity and effectiveness of the DMBE concept can be tested by joint exercises (JEs), simulating disaster events; they can also help reveal additional EIS data interoperability problems. JEs will assess the resilience of DMTFs in various scenarios, with appropriate corrective action taken to improve DMCN members' preparedness and DMTF agility.

\section{CONCLUSIONS AND FURTHER WORK}

As the world climate is changing, mankind is experiencing more frequent and intense catastrophic events. The DM effort must adapt to these changes by addressing urgent cooperation issues in advance and in a holistic manner so that when disaster events 


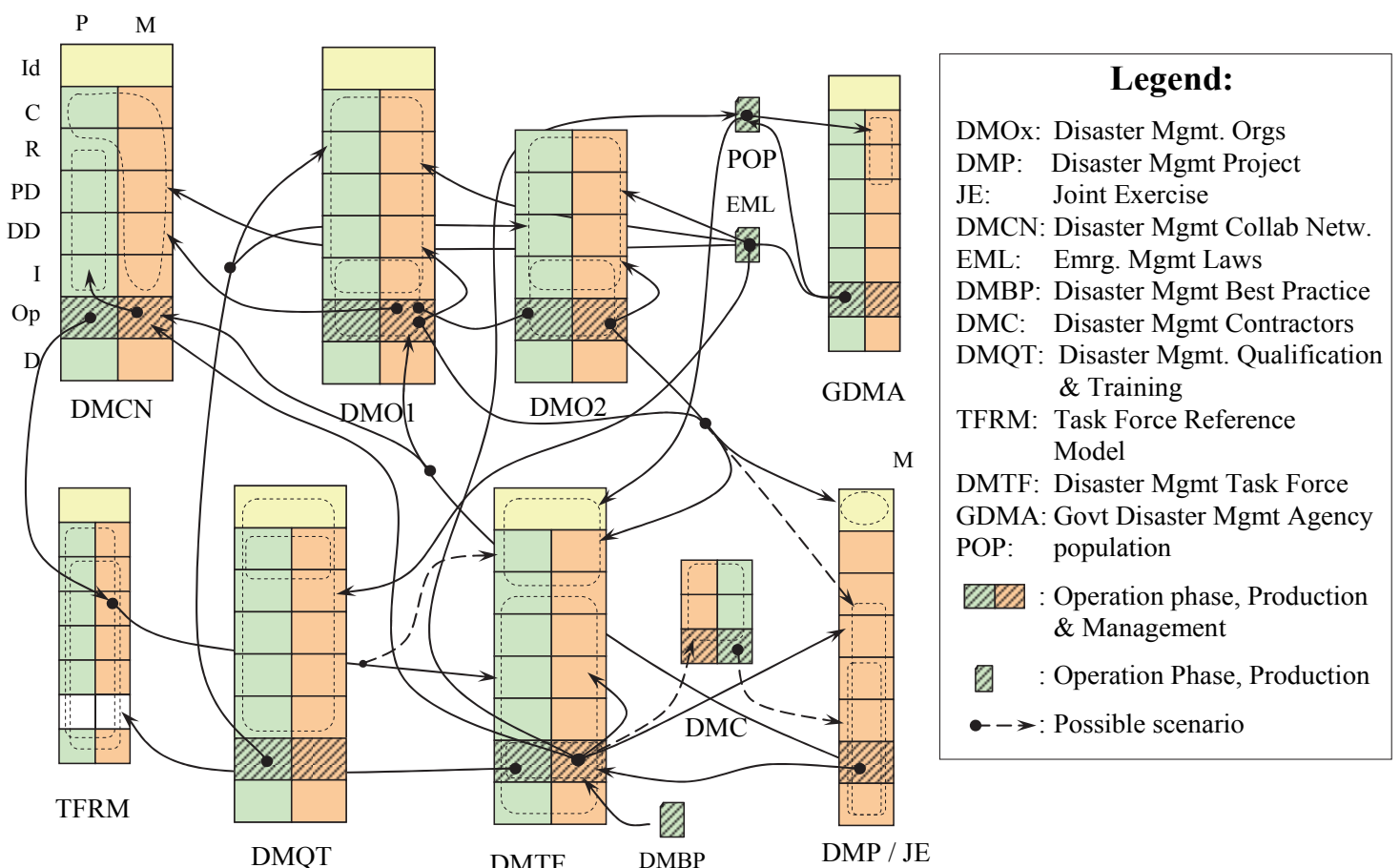

Life cycle phases: Id: Identification; $\mathrm{C}=$ concept; $\mathrm{R}=$ requirements, $\mathrm{PD}=$ preliminary design, $\mathrm{DD}=\mathrm{detailed}$ design,

$\mathrm{I}=$ implementation, $\mathrm{Op}=$ operation, $\mathrm{D}=$ decommissioning. Other aspects: $\mathrm{P}=$ Production / Service, $\mathrm{M}=$ management

Figure 5: High-level IS Data Interoperability requirements in the context of a Disaster Management Collaborative Network and Disaster Management Task Force solution (some details omitted for clarity).

occur, efficient collaborative task forces can be promptly put together. In order to tackle the EIS interoperability problems that plague the DMOs nowadays, it is proposed to adopt a customised collaborative approach based on commercial $\mathrm{CN}$ and VOs.

As DMCN participants' life cycles play a central role in their EIS interoperability, it is argued for and shown how an EA approach and artefacts can provide an integrated, holistic and life cycle-based approach supporting the proposed collaborative paradigm applied to the DM effort.

Further research will concentrate on testing, verifying and validating the findings with DMOs, including the set up of pilot DMCNs future case studies.

\section{REFERENCES}

't Hart, Paul, Boin, Arjen, Stern, Eric, \& Sundelius, Bengt. (2005). The Politics of Crisis Management: Public Leadership under Pressure. Cambridge UK: Cambridge University Press.

Australian Government. (2011). Attorney's General's Office - Emergency Management in Australia Retrieved Mar 30, 2011, from http://www.ema.gov.au/
Brewin, Bob. (2011). Tsunami response reveals poor radio interoperability Retrieved April, 2012, from http://www.nextgov.com/nextgov/ng_20110415_3972. php

Camarinha-Matos, L, Afsarmanesh, H., Galeano, N, \& Molina, A. (2009). Collaborative networked organizations - Concepts and practice in manufacturing enterprises. Computers and Ind. Engineering, 57(1), 46-60.

Chen, D. (2006). Framework for Entrerprise Interoperability Retrieved July, 2011, from http://www.finescluster.eu/fines/jm/Download-document/53-Framew ork-for-Enterprise-Interoperability-Chen.html

Clark, J. L. (2006). Practical aspects of federalizing disaster response. Critical Care, 10, 107-113.

Crawford, Susan. (2012). Why Cell Phones Went Dead After Hurricane Sandy Retrieved February, 2013, from http://www.bloomberg.com/news/2012-11-15/whycell-phones-went-dead-after-hurricane-sandy.html

DoD Architecture Framework Working Group. (2004). DoD Architecture Framework Ver 1.0 Retrieved Feb 2007, 2007, from http://www.dod.mil/cio-nii/docs/Do DAF_v1_Volume I.pdf, Volume II.pdf

Ellis, S., Kanowski, P., \& Whelan, R. (2004). National inquiry into bushfire mitigation and management. Canberra.Retrieved from http://www.ema.gov.au/; www/emaweb/emaweb.nsf/Page/Volunteers2

Esmond, July. (2011). Report on The Attraction, Support and Retention of Emergency Management Volunteers Retrieved Mar 30, 2011, from http://www.ema.gov.au/ 
; www/emaweb/emaweb.nsf/Page/Volunteers2

Federal Emergency Management Agency. (2011). National Response Framework Retrieved Mar 30, 2011, from http://www.fema.gov/pdf/emergency/nrf/about_nrf.pdf

Gartner Research. (2012). IT Glossary, 2012, from http://www.gartner.com/technology/it-glossary/enter prise-architecture.jsp

Government of Queensland. (2012). Department of Community Safety - Structure Retrieved Apr, 2012, from http://www.emergency.qld.gov.au/about/

Government of South Australia. (2004). Emergency Management Act 2004 Retrieved Mar 30, 2011, from http://www.legislation.sa.gov.au/LZ/C/A/EME RGENCY MANAGEMENT ACT 2004.aspx

Heart, F., McKenzie, A., McQuillian, J., \& Walden, D. (1978). ARPANET Completion Report (4799). Burlington, MA.

Igarashi, Y., Kong, L., Yamamoto, L., \& McCreery, C. S. (2011). Anatomy of Historical Tsunamis: Lessons Learned for Tsunami Warning. Pure Applied Geophysics(168), 2043-2063.

Iivari, Juhani. (1991). A Paradigmatic Analysis of Contemporary Schools of IS Development. Eur. J. Information Systems, 1(4), 249-272.

ISO. (2005). ISO14258 Industrial Automation Systems Concepts and Rules for Enterprise Models.

ISO/IEC. (2005). Annex C: GERAM ISO/IS 15704:2000/Amd1:2005: Industrial automation systems - Requirements for enterprise-reference architectures and methodologies.

Kapucu, Naim, Arslan, Tolga, \& Demiroz, Fatih. (2010). Collaborative emergency management and national emergency management network. Disaster Prevention \& Management, 19(4), 452-468.

Keen, P. G. W., \& Scott Morton, M. (1978). Decision Support Systems: An Organisational Perspective. Reading, Massachussetts: Addison-Wesley.

Lehtinen, E., \& Lyytinen, K. (1986). Action based model of information system. Information Systems Research, 11(4), 299-317.

McGregor, D. (1960). The Human Side of Enterprise. New York: McGraw-Hill.

McLennan, Jim. (2008). Issues Facing Australian Volunteer-Based Emergency Services Organisations: 2008 2010 Retrieved Mar 30, 2011, from http://www.ema.gov.au/www/emaweb/emaweb.nsf/Pa ge/Volunteers2

Mo, John, (2007). The use of GERAM for Design of a Virtual Enterprise for a Ship Maintenance Consortium. In Pallab Saha (Ed.), Handbook of Enterprise Systems Architecture in Practice (pp. 351-366). Hershey, USA: IDEA Group.

NATO. (2006). Interoperability for joint operations Retrieved July, 2011, from http//www.nato.int/docu/inter operability/interoperability.pdf

Noran, O., (2008). A Meta-methodology for Collaborative Networked Organisations: Creating Directly Applicable Methods for Enterprise Engineering Projects. Saarbrücken: VDM Verlag Dr. Müller.

Noran, O., (2009). Engineering the Sustainable Business:
An Enterprise Architecture Approach. In Gary Doucet, John Gotze \& Pallab Saha (Eds.), Coherency Management: Architecting the Enterprise for Alignment, Agility, and Assurance (pp. 179-210): International Enterprise Architecture Institute.

OASIS. (2005). Common Alerting Protocol v1.1, 2012, from http://www.oasis-open.org/committees/downlo ad.php/15135/emergency-CAPv1.1-Corrected_DOM.p df

Pava, C. (1983). Managing New Office Technology, An Organisational Strategy. New York: Free Press.

Prizzia, Ross, \& Helfand, Gary. (2001). Emergency preparedness and disaster management in Hawaii. Disaster Prevention and Management, 10(3), 163-172.

Queensland Floods Commission of Enquiry. (2011). Submissions to the Enquiry Retrieved July, 2011, from http://www.floodcommission.qld.gov.au/submissions/

Queensland Government. (2011). Queensland Combined Emergency Services Academy (QCESA) Retrieved Mar 30,2011,from http://www.safecom.sa.gov.au/sit e/emergency management

Stohr, E, \& Konsynsky, B. R. (1992). Information Systems and Decision Processes. California.

Trakas, Athina. (2012). Interoperability - A key requirement for emergency and disaster management, 2012, from http://www.un-spider.org/book/5143/4c-challeng e-communication-coordination-cooperation-capacitydevelopment

United Nations International Strategy for Disaster Reduction Secretariat (UNISDR). (2011). Hyogo Framework for Action 2005-2015: Building the resilience of nations and communities to disasters Retrieved Mar 30, 2011, from http://www.preventionweb.net/files/1037 hyogoframeworkforactionenglish.pdf

Victorian Bushfires Royal Commission. (2009). Submissions to the Enquiry Retrieved July, 2011, from http://www.royalcommission.vic.gov.au/Submissions/ View-Submissions

von Lubitz, Dag K. J. E. , Beakley, James E., \& Patricelli, F. (2008). Disaster Management: The Structure, Function, and Significance of Network-Centric Operations. Journal of Homeland Security and Emergency Management, 5(1), Art 42.

Whitman, L., \& Panetto, H. (2006). The Missing Link: Culture and Language Barriers to Interoperability. Annual Reviews in Control, 30(2), 233-241.

Wickramasinghe, N, \& von Lubitz, Dag K. J. E., (2007). Knowledge Based Enterprises: Theories and Fundamentals (Vol. 5). Hershey PA: IGP Publishing Group.

Wiese, C. R. (2006). Organizing Homeland Security after Katrina: is adaptive management what's missing? Public Administration Review, 66(3), 302-318. 